\title{
Bilateral atypical femur fracture after prolonged use of alendronate - a case report
}

\begin{abstract}
The use of bisphosphonates is among the most agreed upon and widespread therapies for osteoporosis control, with statistically confirmed results showing a decrease in bone resorption. However, there are significant reports of atypical femoral fractures observed in patients with long-term therapy. This study reports a bilateral femoral fracture in a patient after 7 years of treatment with Alendronate.
\end{abstract}

Keywords: Atypical femoral fractures, Alendronate, Biphosphonate

\author{
Volume 9 Issue 4 - 2017 \\ Carlos Roberto Schwartsmann,' Bernardo \\ Vaz Peres Alves, ${ }^{2}$ Gabriel Severo da Silva, ${ }^{2}$ \\ João Pedro Farina Brunelli \\ 'Orthopaedic Professor, Complexo Hospitalar, Santa Casa de \\ Porto Alegre, Brazil \\ ${ }^{2}$ Orthopaedic Assistent, Complexo Hospitalar, Santa Casa de \\ Porto Alegre, Brazil
}

Correspondence: Carlos Roberto Schwartsmann,
Orthopaedic Professor, Complexo Hospitalar, Santa Casa de
Porto Alegre, Brazil, Email schwartsmann@santacasa.tche.br

Received: October 16, 2017 | Published: December 14, 2017

\section{Introduction}

Osteoporosis is an increasingly frequent problem among developed populations due to the longer life expectancy we experience nowadays. Anti-resorptive drugs, such as bisphosphonates, are among the most frequently used pharmaceutical treatments for osteoporosis. ${ }^{1}$ It is agreed upon that the absolute number of vertebral and nonvertebral fractures decreases greatly with this therapy. ${ }^{1}$ However, paradoxically, attention is brought to the increased number of atypical femoral fractures associated with low-energy trauma after long-term bisphosphonate treatment, alendronate being the most commonly used. The time taken for a significant increase in atypical fractures associated with alendronate to occur is not yet clear, but some data suggest 5 years, ${ }^{2}$ although there are reports of fractures following an even shorter duration of therapy..$^{2-17}$ The explanation consists of excessive suppression of osteoclastic activity and a consequent decrease in bone remodeling. ${ }^{2-5}$

\section{Case report}

We report a case of bilateral femoral fracture in a patient after 7 years of treatment with alendronate at a dose of $70 \mathrm{mg}$ weekly. The patient was a 67 y.o. female with no history of any comorbidity such as diabetes, alcohol abuse, smoking, chronic use of glucocorticoids or others. She fell from her own height in March 2014, resulting in a transverse femoral shaft fracture of the left femur (Figure 1A), treated with a blocked anterograde intramedullary nail (Figure 1B \& 1C). In June 2016, she suffered a contralateral short oblique femoral fracture with no history of direct trauma, presumably with a torsional mechanism - rotation around the body axis (Figure 2A). The treatment of choice was also a blocked anterograde intramedullary nail (Figures 2B \& 2C). We can observe in Figures $1 \& 2$ the absence of compromised trabecular bone or thinning of cortical bone, corroborating the probable pathophysiology behind bisphosphonateassociated fractures - decreased bone remodelling - and not osteopenia/ osteoporosis.

\section{Discussion}

The use of bisphopshonates in the treatment of osteoporosis has wide scientific support in the literature, with benefits overcoming the risks even with a treatment period longer than 10 years. ${ }^{4,5}$ However, therapy longer than 5 years and poor adherence to treatment are associated with a higher number of atypical femoral fractures. When analysed in the context of all femoral shaft fractures, atypical fractures may be responsible for up to $50 \%$ of them, ${ }^{7}$ depending on the population studied. Bilateral commitment is described in up to $30 \%$ of these patients, especially with a treatment period longer than 3 years. ${ }^{12,16}$ Moreover, shaft fractures are more frequent than subtrochanteric fractures in patients older than 50 years. ${ }^{6,12,14}$ The prevalence of female patients with femoral fractures - due to the increased incidence of osteoporosis is notable, especially those with atypical patterns, which are responsible for up to $95 \%$ of cases in this group of patients. ${ }^{7}$ The most accepted ethiopathogeny is excessive suppression of osteoclastic activity and a consequent decrease in bone remodelling, leading to cortical hypermineralization, increased rigidity and a subsequent decrease in flexibility and resistance..$^{2-5}$ Radiographic findings are thickened cortical bone and complete transverse and short-oblique non-comminuted fractures comprising mostly the subtrochanteric region and the diaphysis. ${ }^{9}$ There are also reports of impacted or incomplete fractures - occurring in approximately $10 \%$ of cases ${ }^{7}-$ without history of trauma, which can make the diagnosis difficult. In these cases is important to obtain contralateral radiographs and other complementary exams, such as CT and MRI scans, to adequately diagnose and stage the lesion. ${ }^{10}$ These cases require the suspension of bisphosphonates and maintenance of vitamin D and calcium supplementation combined with prophylactic surgical treatment. ${ }^{11,12}$

The most often used methods of surgical fixation for both prophylactic and fractured cases are blocked intramedullary nailing and plating (DCP/LC-DCP) in diaphyseal fractures and proximal femoral nailing and DHS/DCS plates for subtrochanteric patterns, chosen according to the surgeon's experience and preference. ${ }^{14,17}$ Our team preferred blocked intramedullary anterograde nailing based on the best results observed in the literature in comparison with plating ${ }^{10}$ for allowing early gait training and for being less invasive: important aspects of improving consolidation in these cases, which already have decreased bone remodelling. The patient in this study presented full radiographic consolidation after a mean of 6.8 months, similar to the time of 7.3 months found in the literature. ${ }^{10}$ It is important to note the tendency of these fractures to develop delay in consolidation and pseudoarthrosis in up to $50 \%$ of cases. ${ }^{13-15}$ 


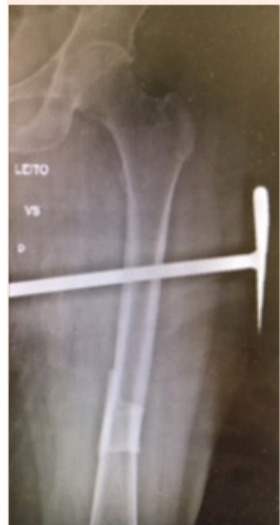

A

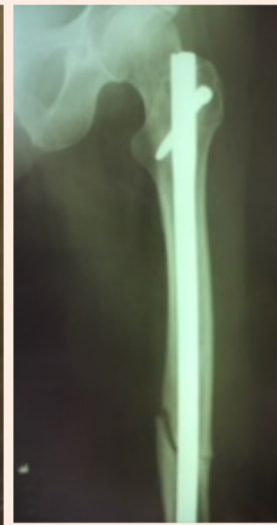

B

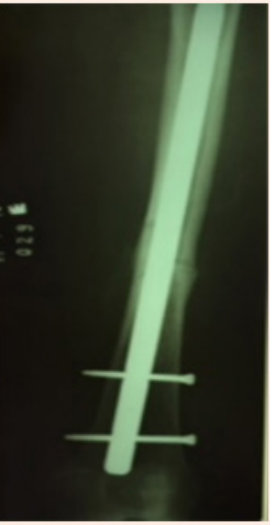

C
Figure IA Left femoral anteroposterior radiograph - Simple short oblique tract fracture with cortical thickening. IB \& IC: Postoperative radiograph anterograde blocked nail left femur.

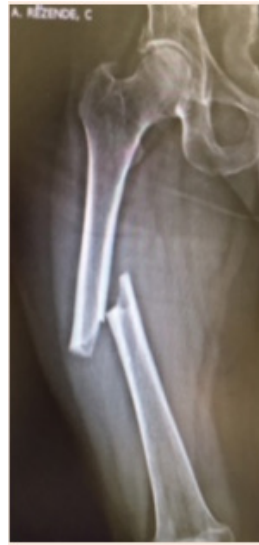

A

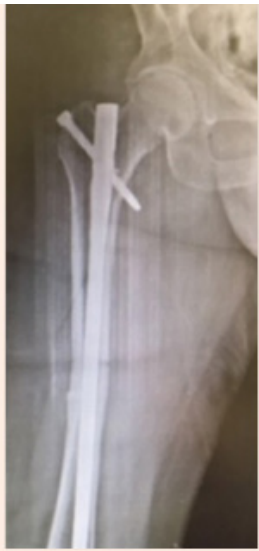

B

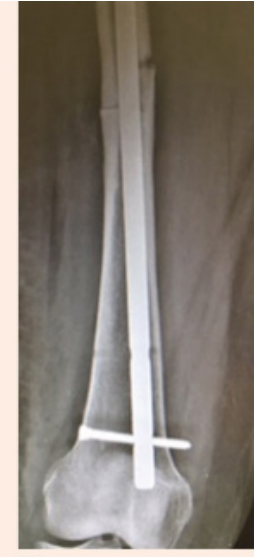

C

\section{Conflicts of interest}

None.

\section{References}

1. Black DM, Rosen CJ. Clinical Practice. Postmenopausal Osteoporosis. $N$ Engl J Med. 2016;21:374(3):254-262.

2. Adler RA. Bisphosphonates and atypical femoral fractures. Curr Opin Endocrinol Diabetes Obes. 2016;23(6):430-434

3. Balach T, Baldwin PC, Intravia J. Atypical Femur Fractures Associated With Diphosphonate Use. J Am Acad Orthop Surg. 2015;23(9):550-557.

4. Abrahamsen B. Risk of hip, subtrochanteric, and femoral shaft fractures among mid and long term users of alendronate: nationwide cohort and nested case-control study. BMJ. 2016;28(353):i3365.

5. Nelson B Watts. Long-term risks of bisphosphonate therapy. Arq Bras Endocrinol Metab. 2014;58(5):523-529.

6. Wolin EA, Banks KP, Vroman PJ. Bisphosphonate-associated atypical subtrochanteric femur fracture. J Nucl Med Technol. 2015;43(1):72-73.

7. Patel RN, Ashraf A, Sundaram M. Atypical Fractures Following Bisphosphonate Therapy. Semin Musculoskelet Radiol. 2016;20(4):376-381.

8. Ballas EG, Mavrogenis AF, Karamanis E, et al. Low-energy femoral shaft fractures after long-term alendronate therapy: report of seven cases. Eur J Orthop Surg Traumatol. 2015;25(1):181-187.

9. Park-Wyllie LY, Mamdani MM, Juurlink DN, et al. Bisphosphonate use and the risk of subtrochanteric or femoral shaft fractures in older women. JAMA. 2011;305(8):783-9.

10. Liu L, Li C, Yang P, et al. Association between alendronate and atypical femur fractures: a meta-analysis. Endocr Connect. 2015;4(1):58-64.

11. Kohli R, Siva C.Atypical Femoral Fractures Can Happen Even With Short Duration Bisphosphonate Treatment. Conn Med. 2015;79(7):419-422.

12. Kondo N, Yoda T, Fujisawa J, et al. Bilateral atypical femoral subtrochanteric fractures in a premenopausal patient receiving prolonged bisphosphonate therapy: evidence of severely suppressed bone turnover - Clin Cases Miner Bone Meta. 2015;12(3):273-277. tract fracture with cortical thickening. 2B \& 2C Postoperative radiography anterograde blocked nail right femur.

\section{Conclusion}

As possible measures to prevent atypical femoral fractures in patients treated with bisphosphonates, we recommend orientation regarding treatment adherence, periodical medical consultations and limiting the use of bisphosphonates to a maximum of 5 years. ${ }^{16}$ Once the fracture is stabilised, we suggest discontinuation of treatment and proper fixation of the fracture. Considering the high incidence of bilateral fractures, it is necessary to give special attention to the contralateral femur to identify adequate candidates for prophylactic surgery.

\section{Acknowledgements}

None.
13. Schilcher J, Koeppen V, Aspenberg P, et al. Risk of atypical femoral fracture during and after bisphosphonate use. Acta Orthop. 2015;86(1):100-107.

14. Bhadada SK1, Sridhar S, Muthukrishnan J, et al. Predictors of atypical femoral fractures during long term bisphosphonate therapy: a case series \& review of literature. Indian J Med Res. 2014;140(1):46-54.

15. Pedrazzoni M, Giusti A, Girasole G, et al. Atypical femoral fractures in Italy: a retrospective analysis in a large urban emergency department during a 7-year period. J Bone Miner Metab . 2016.

16. Szolomayer LK, Ibe IK, Lindskog DM. Bilateral atypical fêmur fractures without bisphosphonate exposure. Skeletal Radiol. 2016;46(2):241-247.

17. Bubbear JS. Atypical Femur Fractures in Patients Treated with Bisphosphonates: Identification, Management, and Prevention. Rambam Maimonides Med. 2016;J7(4). 